\title{
Analysis of Uncertain Data: Selection of Probes for Information Gathering
}

\author{
Anatole Gershman \\ anatoleg@cs.cmu.edu \\ www.cs.cmu.edu/ anatoleg
}

\author{
Eugene Fink \\ e.fink@cs.cmu.edu \\ www.cs.cmu.edu/ eugene
}

\author{
Bin Fu \\ binf@cs.cmu.edu \\ www.cs.cmu.edu/ binf
}

\author{
Jaime G. Carbonell \\ jgc@cs.cmu.edu \\ www.cs.cmu.edu/ jgc
}

\section{Computer Science, Carnegie Mellon University, Pittsburgh, PA 15213, United States}

\begin{abstract}
We consider the problem of gathering data for evaluation of given hypotheses, and describe a method for analyzing tradeoffs between the expected utility and the cost of data collection.
\end{abstract}

Keywords-Uncertainty, hypothesis evaluation, data gathering, Bayesian reasoning, artificial intelligence.

\section{INTRODUCTION}

$\mathrm{W}$ HEN estimating the likelihood of given hypotheses, we sometimes have to base the analysis on limited data. If available data are insufficient, we need to gather more information; this gathering may be costly, and we often have to evaluate tradeoffs between its utility and costs. For instance, when a physician diagnoses a patient's illness, she sometimes faces the question whether to order more medical tests, which may be painful for the patient and incur significant expenses. Similarly, when a military analyst tries to anticipate actions of an adversary, she may have to decide which resources to use for intelligence gathering.

We have investigated several related problems and developed a suite of techniques for representation of insufficient data [Bardak et al., 2006a; Fu et al., 2008]; reasoning and hypothesis evaluation based on limited data [Fink et al., 2006; Gershman et al., 2009]; and planning of additional data collection [Bardak et al., 2006b].

We report one of the developed techniques for information gathering, which is focused on probing an observed system by external actions and watching its response. For instance, a physician may "probe" a patient by asking him to run on a treadmill, and then observe how this exercise affects electrocardiogram. As another example, a military analyst may suggest political or economic actions against an adversarial government and observe its response. The challenge in such scenarios is to select the right actions from an available arsenal; that is, optimize the expected value of probing.

We first give an example scenario (Section II) and formalize the general problem (Sections III and IV). We then describe a technique for selecting probes (Sections IV-VI) and give experiments on its effectiveness (Section VII).

The manuscript was received on March 31,2009. The described work was supported by the Air Force Research Laboratory (AFRL) under Contract No. FA8750-07-2-0137.

\section{EXAMPLE}

We consider the task of a business analyst who is observing a small pharmaceutical company and trying to infer the plans of its management. The company has recently advertised a new medication and announced that its main focus is on expanding its sales. The analyst however suspects that the company is also working on another new medication, which has a potential for greater sales. If she is right, the company is developing this second medication in secrecy, as it does not want to reveal its plans to competitors. The analyst has to distinguish between two hypotheses:

- The company focuses exclusively on expanding the sales of the announced product.

- The company puts significant resources into the development of another product.

She is evaluating these scenarios based on public data, possibly augmented with private knowledge gained from her friends in pharmaceutical industry.

We have recently published a heuristic technique for this task, which allows distinguishing among given hypotheses based on limited data even when these data are insufficient for a rigorous statistical analysis [Gershman et al., 2009]. It may happen, however, that the data are insufficient even for approximate heuristics. Then, the analyst has to gather additional observations.

We consider one specific type of data gathering, based on affecting the company by external actions, called probes, and observing its response. We assume that the analyst has resources for putting some pressure on the company; for example, she may affect the price of the company's product or try to hire away its chief scientist. If she applies such probes, the company's reaction may reveal its plans. For instance, if the company is working on a new medication, it is likely to exhibit a lot of concern about keeping its scientists but less concern about the price of its current product.

\section{GENERAL PROBLEM}

To formalize the probe-selection task, suppose that the analyst has to distinguish among mutually exclusive hypotheses, denoted $H_{1}, H_{2}, \ldots, H_{n}$. She can observe several features of the company, denoted $o b s_{1}, o b s_{2}, \ldots, o b s_{m}$, where each observa- 
tion is a variable that takes one of several discrete values. She has several probing actions at her disposal, probe $_{1}, \ldots$, probe $_{k}$; one of them may represent passive data collection without affecting the company, whereas the others involve active probing. Suppose further that the analyst has the following information about prior probabilities, probe costs, possible observations, and expected probing results.

- Prior probabilities: For each hypothesis, the analyst knows its prior probability. Thus, she has an array of $n$ priors, denoted prior [1..n]; the sum of these priors is 1.0.

- Probe costs: For each probe, the analyst knows its expected cost; thus, she has an array of $k$ numeric costs, denoted $\operatorname{cost}[1 . . k]$.

- Possible observations: For every observation $o b s_{a}$, the analyst knows its possible values, as well as the overall number of these values, denoted num [a]; that is, she knows the domain of the variable $o b s_{a}$. Thus, she has an array of $m$ integers, denoted num[1..m], that represents the number of possible values for each of the $m$ observations.

- Observation distributions: The likelihood of specific observed values depends on (1) which hypothesis is correct and (2) which probe has been applied. For every hypothesis and every probe, the analyst knows the related probability distribution of each observation. Thus, she has a three-dimensional array with $n \cdot m \cdot k$ elements, denoted chance $[1 . . n, 1 . . m, 1 . . k]$, where each element is a probability density function of possible observations. Every element chance $[i, a, j]$ of this array is itself a one-dimensional array with num $[a]$ elements, which represent the probabilities of possible values of $o b s_{a}$. That is, chance $[i, a, j][b]$ is the likelihood of observing the $b$ th value of $o b s_{a}$ after applying probe $_{j}$, in case if hypothesis $H_{i}$ is correct.

- Observation probabilities: When the analyst applies a probe, some previously observable features may become "invisible," for example, due to the resulting changes in the company security policies. If a feature becomes unobservable, the analyst cannot use it in hypothesis evaluation. The chances that a feature remains observable depend on (1) which hypothesis is correct and (2) which probe has been applied. Thus, the analyst has a three-dimensional array with $n \cdot m \cdot k$ elements, vis-chance $[1 . . n, 1 . . m, 1 . . k]$, where each element is the probability of observing a specific feature, given a specific probe and hypothesis. That is, vis-chance $[i, a, j]$ is the likelihood that $o b s_{a}$ remains visible after applying probe $_{j}$, in case if hypothesis $H_{i}$ is correct.

Note that we assume the availability of all prior information in the above list, but we do not assume its perfect accuracy. The developed technique works even if most of the prior knowledge is based on rough estimates. Gathering of the prior information requires different approaches in different domains. The analyst may gain it from past experience, expert advice, empirical studies, or computational models of alternative future developments.

The analyst has to use this knowledge in selecting the most appropriate probe. After applying the selected probe, she will collect new observations and use them in evaluating the posterior probability of each given hypothesis.

\section{UTILITY FUNCTION}

We define quantitative probe gain, which represents the expected utility of obtaining more data and improving probability estimates. Intuitively, the importance of hypothesis evaluation depends on its intended use, that is, its impact on future actions [Grünwald and Dawid, 2004]. For example, if the analyst watches the pharmaceutical company just out of personal curiosity, she probably would not consider any probing. On the other hand, if she works for an investment firm that plans a major purchase of the company stock, then the accuracy of her conclusions is essential for the firm's profit, and she may be willing to use costly probing.

To formalize this intuition, suppose that hypothesis evaluation affects certain strategy, such as investment into the company, and the analyst has to select among alternative strategies, $S_{1}, S_{2}, \ldots, S_{l}$. Suppose further that she knows the expected value of each strategy for each possible scenario.

- Strategy utilities: The utility of a strategy depends on which hypothesis is correct. For every hypothesis, the analyst knows the expected value of each strategy under this hypothesis. Thus, she has a two-dimensional matrix with $n \cdot l$ elements, denoted $u t i l[1 . . n, 1 . . l]$, where each element is a strategy utility. That is, $u t i l[i, c]$ is the utility of strategy $S_{c}$ in case if hypothesis $H_{i}$ is correct. For example, it may represent the estimated growth of the company stock in case if it develops a new medication.

If estimated hypothesis probabilities are prior $[1 . . n]$, then the expected utility of a specific strategy $S_{c}$ is

$$
\operatorname{utility}\left(S_{c}\right)=\operatorname{prior}[1] \cdot \operatorname{util}[1, c]+\ldots+\operatorname{prior}[n] \cdot u t i l[n, c] .
$$

The best choice is the strategy $S_{\text {good }}$ with the maximal expected utility.

Now suppose that the analyst uses some probe, say probe $_{j}$, collects additional observations, and gets a posterior estimate of hypothesis probabilities, post $[1 . . n]$. She may now select a new strategy, $S_{\text {better }}$, based on this new estimate. Thus, the use of probe $_{j}$ has resulted in replacing $S_{\text {good }}$ with $S_{\text {better }}$; the expected utility increase due to this replacement, based on the new knowledge obtained through the use of $p$ robe $e_{j}$, is the utility of the probe:

$$
\begin{aligned}
& \text { probe-util( } \left.\text { probe }_{j}\right) \\
& =\operatorname{post}[1] \cdot(\text { util }[1, \text { better }]-\text { util }[1, \text { good }]) \\
& +\ldots+\operatorname{post}[n] \cdot(u t i l[n, \text { better }]-u t i l[n, \operatorname{good}]) \text {. }
\end{aligned}
$$

The overall gain is the difference between the utility and the probe cost:

$$
\operatorname{gain}\left(\operatorname{prob}_{j}\right)=\operatorname{prob}-u t i l\left(\operatorname{probe}_{j}\right)-\operatorname{cost}[j] \text {. }
$$

When the analyst plans her probing, she has to evaluate the expected gain of every available probe. If all probes have negative expectations, she should not do any probing; else, she should use the probe with the greatest expected gain.

To compare this utility model with the classical information gain, we consider a rather artificial example of an investment decision. Suppose that we have two hypotheses, 
$H_{1}$ and $H_{2}$, about the company's plans, which completely cover the set of all possibilities. We intend to invest a certain fraction $t$ of available funds into the company, and we are deciding on the value of $t$, which is between 0.0 and 1.0. We further suppose that, if $H_{1}$ is correct, the expected return is $\log t$; else, it is $\log (1-t)$.

If the probability estimate for $H_{1}$ is prior [1], the expectation of the investment return is

$$
\operatorname{utility}(t)=\operatorname{prior}[1] \cdot \log t+(1-\operatorname{prior}[1]) \cdot \log (1-t) .
$$

The optimal investment is $t=$ prior [1], which maximizes the above expression. Thus, the optimal expected return is

$$
\text { prior }[1] \cdot \log \text { prior }[1]+(1-\text { prior }[1]) \cdot \log (1-\text { prior }[1]),
$$

which is the negation of the classical Shannon's entropy [Shannon, 1948].

If we use some probe and obtain a better probability estimate $\operatorname{post}[1]$, which would lead to investing $t=\operatorname{post}[1]$, then the resulting utility increase is

$$
\begin{aligned}
& \text { probe-util(probe) } \\
& \quad=\operatorname{post}[1] \cdot \log \operatorname{post}[1]+(1-\operatorname{post}[1]) \cdot \log (1-\operatorname{post}[1]) \\
& \quad-\operatorname{post}[1] \cdot \log \operatorname{prior}[1]-(1-\operatorname{post}[1]) \cdot \log (1-\operatorname{prior}[1]) .
\end{aligned}
$$

If the probing cost is zero, this expression represents the expected probe gain. It is identical to the definition of the Kullback-Leiber divergence between the initial and the improved probability estimates [Kullback and Leibler, 1951; Kullback, 1987], which is also known as information gain.

This artificial example shows that the proposed definition of the probe gain is a generalization of the classical information gain. The key difference from the classical definition is that we account for (1) the probe cost and (2) the utilities of possible strategies, which may be different from the information utility in Shannon's expression.

In practical situations, we need to obtain probe-cost and strategy-utility estimates from domain experts. If we cannot get expert advice, we use Shannon's function, which is the default utility in the implemented system.

\section{Probe eValuation in a ONE-Feature SCEnario}

We now explain a procedure for estimating probe gains. First, we consider a simplified scenario, in which the analyst can observe only one feature $o b s_{a}$ of the company, and has to identifying the probe that will maximize the value of her observation. Then, in Section VI, we analyze the case of multiple observations.

If the analyst had to base her strategy selection on the prior probabilities, without any probing, she would pick the strategy $S_{\text {good }}$ that maximized the following expression:

$$
\operatorname{utility}\left(S_{c}\right)=\operatorname{prior}[1] \cdot u t i l[1, c]+\ldots+\operatorname{prior}[n] \cdot u t i l[n, c] .
$$

On the other hand, if she applied some probe, $p r o b_{j}$, and afterward observed that $o b s_{a}$ had taken its $b$ th value, then she could use the Bayesian rule to compute the array post $[1 . . n]$ of posterior probabilities.

To obtain these posteriors, she would first calculate the integrated likelihood (also called marginal likelihood) of observing the $b$ th value of $o b s_{a}$, by summing its chances under all possible hypotheses after applying probe $_{j}$ :

$$
\begin{aligned}
& \text { likelihood }(b) \\
& =\operatorname{chance}[1, a, j][b] \cdot \operatorname{prior}[1] \\
& \quad+\ldots+\operatorname{chance}[n, a, j][b] \cdot \operatorname{prior}[n],
\end{aligned}
$$

and she would then use it to determine the posterior probability of each hypothesis $H_{i}$ :

$$
\begin{aligned}
& \text { post }[i]=\operatorname{probability}\left(H_{i} \mid \text { bth value of } \operatorname{obs}_{a}\right) \\
& \quad=\operatorname{chance}[i, a, j][b] \cdot \operatorname{prior}[i] / \operatorname{likelihood}(b) .
\end{aligned}
$$

Note that the resulting posteriors depend on the observed value of $o b s_{a}$. Formally, it means that the array post $[1 . . n]$ of posteriors is a function of $b$. We can write this function as $\operatorname{post}(b)$, and denote its $i$ th element by post $(b)[i]$.

After computing these posteriors, the analyst would select the strategy $S_{\text {better }}$ that maximized the following expression over possible values of the strategy index $c$ :

$u t i l i t y\left(S_{c}\right)=\operatorname{post}(b)[1] \cdot u t i l[1, c]+\ldots+\operatorname{post}(b)[n] \cdot u t i l[n, c]$.

Note that the choice of this optimal strategy depends on the observed value of $o b s_{a}$. Thus, the selected index "better" is a function of $b$, and we can write it as $\operatorname{better}(b)$.

The gain due to applying $p r o b_{j}$ and then selecting a better strategy, $S_{\text {better }(b)}$, would be the difference between the resulting utility increase and the cost of prob $_{j}$ :

$$
\begin{aligned}
& \operatorname{probe-gain}(b) \\
& =\operatorname{post}(b)[1] \cdot(u t i l[1, \operatorname{better}(b)]-u t i l[1, \operatorname{good}]) \\
& \quad+\ldots+\operatorname{post}(b)[n] \cdot(u t i l[n, \operatorname{better}(b)]-u t i l[n, \operatorname{good}]) \\
& \quad-\operatorname{cost}[j] .
\end{aligned}
$$

We can evaluate the expected gain of probe $_{j}$ by averaging this expression over all possible values of $o b s_{a}$ :

$$
\begin{aligned}
& \text { likelihood }(1) \cdot \operatorname{probe-gain}(1) \\
& \quad+\ldots+\text { likelihood }(\text { num }[a]) \cdot \operatorname{probe-gain}(\text { num }[a]) .
\end{aligned}
$$

The analyst also has to account for the possibility that $o b s_{a}$ may become invisible due to the probing; then, she would end up paying for the probe but getting no data. The chances that $o b s_{a}$ remains observable are vis-chance $[i, a, j]$, which leads to the following expression for the expected probe gain:

$$
\begin{aligned}
& \text { single-obs-gain( } \text { probe }_{j} \text { ) } \\
& =\text { vis-chance }[i, a, j] \\
& \text { - (likelihood (1) - probe-gain (1) } \\
& +\ldots+\operatorname{likelihood}(\text { num }[a]) \cdot \operatorname{probe}-g a i n(\text { num }[a])) \\
& +(1.0-v i s-\text { chance }[i, a, j]) \cdot \operatorname{cost}[j] \text {. }
\end{aligned}
$$

We have all data required for computing this expression, which means that we can estimate the gain of probe $_{j}$. Note that, although we have not explicitly included the index $j$ in the notation for the likelihood and probe-gain terms of the above expression, these terms depend on $j$, and the earlier expressions for calculating them use $j$. The analyst can thus determine the expected gain for every probe and select the probe with the greatest gain.

\section{Probe eVAluation in a multi-Feature SCEnARio}

If the analyst were to use observations of multiple features in her evaluation, she would have to account for correlations among them, which means that she would need data on their joint probability distribution. Unfortunately, obtaining even rough estimates of joint probabilities is impractically difficult 
in most real-world scenarios. Furthermore, we usually cannot assume that observations are independent, nor can we reliably determine which of them are interdependent.

For instance, different news releases may be related to each other to some unknown degree; different stock performance indicators usually have a high degree of correlation, which cannot be reliably evaluated; and different "independent" experts often repeat each other's opinions.

The history of business analysis and military intelligence is full of spectacular mistakes due to inappropriate uses of the independence assumption. In particular, it has happened time and again that analysts would receive the same inaccurate or falsified data from the same source, through several different channels, and would mistakenly view it as independent reports that corroborate each other.

To avoid this pitfall, we use a simple, very conservative heuristic, which never overestimates the value of available data. Specifically, we identify the most relevant observation and do not use other observations to corroborate it. On the upside, this approach never leads to unjustified excessive confidence; on the downside, it may significantly underestimate the value of observations.

To formalize it, we consider the evaluation of a specific probe, say probe $_{j}$, and suppose that the analyst can observe $m$ company features. For each individual observation $o b s_{a}$, she can use the technique of Section $\mathrm{V}$ to determine what the probe gain would be if she used only $o b s_{a}$; we denote it single-obs-gain $\left(\right.$ probe $\left._{j}, o b s_{a}\right)$. Then, she can identify the observation that maximizes this gain, which is the most relevant observation for probe $_{j}$. The probe gain for this observation is

$$
\begin{aligned}
& \operatorname{gain}_{\left(\text {prob }_{j}\right)} \\
& =\max \left(\operatorname{single-obs-gain}\left(\text { prob }_{j}, o b s_{1}\right), \ldots,\right. \\
& \left.\quad \text { single-obs-gain }\left(\text { prob }_{j}, o b s_{m}\right)\right) .
\end{aligned}
$$

Note that different observations may maximize this expression for different probes, which means that the choice of the most relevant observation depends on the probe. For each available probe, the analyst should identify the most relevant observation and compute the respective gain. The final "winner" is the probe with the greatest gain.

\section{EXPERIMENTS}

We have evaluated the described technique in the context of the PAINT architecture, built by several research teams from different institutions under the PAINT program of IARPA. This architecture is a suite of tools for modeling a partially observable organization, analyzing possible developments within the organization, and planning the collection of additional data. It supports modeling of the organization's management, decision processes, projects, and resource allocation. We have been responsible for several uncertainty-analysis tools in PAINT, including probe selection.

Experimental design. We have experimented with tasks of determining whether an observed company is secretly working on a new product, similar to the example in Section II. First, we describe the hypotheses, probes, observations, and utility functions. Then, we give the results of applying the system to several specific tasks.
- Hypotheses: We have considered four hypotheses, which give rise to four different organizational models in PAINT.

$\boldsymbol{H}_{1}$ : The company focuses exclusively on the sales of its current product and has no secret agenda.

$\boldsymbol{H}_{\mathbf{2}}$ : The company puts limited resources into its secret development of a new product, but its main focus is the sales of its current product.

$\boldsymbol{H}_{\mathbf{3}}$ : The company puts major resources into its new-product development; it views the current sales as an important secondary activity.

$\boldsymbol{H}_{4}$ : The company focuses almost exclusively on its new-product development; its sales are just a smokescreen to mislead its competitors.

- Observations: We have considered eighty-four observable features of the modeled company, which represent public data about its sales and known projects. The observables do not include any direct data about the new product, since these data are "invisible" to the public.

- Probes: We have assumed the availability of five probes. The first is the do-nothing "probe," that is, passive data collection without affecting the company; the other four are based on reducing market availability of various resources used by the company. Of course, we have experimented only with simulated probing, and we have not taken any actions in the real-world markets.

- Prior probabilities: We have assumed that all hypotheses are equally likely; that is, they all have the same prior.

- Observation distributions: To obtain probability distributions for observations, we have simulated the company behavior under different scenarios. For every hypothesis and every probe, we have run the PAINT models to predict the likelihood of each possible observed value.

We have experimented with the following two utility models.

- Simple rewards: We get a fixed positive reward (say, one dollar) for choosing the right hypothesis; and no reward (zero dollars) for a wrong guess. In terms of the general model in Section IV, it means that every hypothesis $H_{i}$ requires its unique strategy $S_{i}$. If the selected strategy $S_{i}$ matches the true hypothesis $H_{i}$, its utility is one; if we choose any other strategy $S_{c}$, where $c \neq i$, the utility is zero.

- Shannon utility: The utility is based on Shannon's formula, as defined in the end of Section IV.

We have used the technique of Section VI to choose the most relevant observation for each of these two utilities, and compared it with the following three alternative techniques.

- Jensen-Shannon divergence: We select the most relevant observation based on the Jensen-Shannon divergence [Lin, 1991] among the distributions of observation values under different hypotheses. That is, for each observable variable, we compute the divergence among its predicted probability density functions under different hypotheses, and we select the observation with the greatest expected divergence; we then use it in hypothesis evaluation.

- Randomly chosen observation: We pick an observation at random among the $n$ available observations, and then use it in hypothesis evaluation. Clearly, it is a very ineffective method; we have included it in order to show that the de- 
veloped technique is at all useful, that is, far better than a trivial approach.

- Independence assumption: We evaluate the given hypotheses using the standard Bayesian approach under the assumption that all observations are independent.

To test these techniques, we have considered three different tasks that require hypothesis evaluation and probe selection. We have applied each technique to estimate the posterior probabilities and identify the most likely hypothesis, and we have used the percentage of correctly identified hypotheses as the final performance measure.

Task 1: Differentiation between two hypotheses based on given probing results. We apply one of the probes and then use the system to distinguish between $H_{1}$ and one other hypothesis. In this task, the system does not select a probe; it uses the given probing results.

Since we have five techniques to be evaluated, five probes, and three hypotheses that can be used as alternatives to $H_{1}$, we have experimented with all $5 \cdot 5 \cdot 3=75$ possible settings. For every setting, we have run 2000 Monte Carlo simulations of the PAINT models and then averaged the results. We show the performance in Table I; for each setting, the respective table entry is the percentage of the right answers, that is, correctly identified hypotheses.

Unsurprisingly, the performance based on the random choice of an observation is poor; it is slightly better than the direct random guessing of a hypothesis, which would be $50 \%$ accurate, because the system occasionally picks an observation that is indeed relevant, and then uses it to identify the correct hypothesis.

The performance of the Bayesian approach with the independence assumption is even worse; in fact, it is as bad as the direct random guessing. This result confirms once again that we usually cannot assume the independence of different data sources, and an attempt to use this assumption may lead to grossly incorrect conclusions.

On the other hand, the techniques based on the simple rewards, Shannon utility, and Jensen-Shannon divergence give much better results; the experiments show no difference in the accuracy among these three techniques. Their accuracy of distinguishing $\mathrm{H}_{1}$ from $\mathrm{H}_{2}$ is sometimes low, especially if we use no active probing (see the results for probe $_{1}$, which is the passive do-nothing "probe"); that of distinguishing $H_{1}$ from $H_{3}$ is much better; and that of distinguishing $H_{1}$ from $H_{4}$ is $100 \%$ accurate. The reason is that the hypotheses $H_{1}$ and $H_{2}$ describe similar situations, which are hard to distinguish; $H_{1}$ and $H_{3}$ are less similar; and finally $H_{1}$ and $H_{4}$ are very different, which makes them easy to differentiate.

Task 2: Selection of a probe for differentiation between two hypotheses. We consider the same task of distinguishing between two hypotheses, but we now apply the system to identify the most relevant probe rather than using a given probe. The techniques based on the simple rewards, Shannon utility, and Jensen-Shannon divergence lead to selecting probe $_{2}$ in all cases, which is indeed the right choice. It gives the $99 \%$ accuracy for distinguishing $H_{1}$ from $H_{2}$ or $H_{3}$, and the $100 \%$ accuracy for distinguishing $H_{1}$ from $H_{4}$. On the other hand, the random-observation and the indepen- dence-assumption techniques select probes practically at random, picking each of the five in about $20 \%$ of cases.

Task 3: Differentiation among four hypotheses. We consider the situation when all four hypotheses are equally likely a priori, and run two related tests.

In the first test, the system determines whether $H_{1}$ is true of false; that is, it distinguishes between two hypotheses: $H_{1}$ (with the prior of 0.25 ) and " $H_{2}$ or $H_{3}$ or $H_{4}$ " (with the prior of $0.75)$. In the second test, the system determines which of the four hypotheses is true; that is, it evaluates all four hypotheses and selects the one with the greatest posterior. We show the results of both tests in Table II.

The accuracy of evaluating $H_{1}$ in the first test is relatively high. The system selects either probe $_{2}$ or probe $_{4}$ in all experiments, which leads to $94 \%$ of correct answers.

The accuracy of selecting the most likely among the four hypotheses is of course lower than that in the two-hypothesis case. If we use the simple rewards, the system correctly selects probe $_{2}$, which leads to the $61 \%$ accuracy. If we run the same experiment with the Shannon utility, the system expects near-identical gains for all available probes; it selects the passive do-nothing probe, that is, probe $_{1}$, which is the default tie-breaker in such situations. The resulting percentage of correct answers for the Shannon utility is $49 \%$. Note that it is significantly higher than the accuracy of the direct random guessing, which would be $25 \%$.

Summary. The initial experiments have confirmed that the developed technique is effective for (1) selecting appropriate probes and (2) identifying the correct hypothesis among a small set of candidates. Unsurprisingly, the accuracy declines with the increase in the number of candidate hypotheses, but it remains well above the random guessing. The experiments suggest that the accuracy is insensitive to the choice of a utility function; specifically, the results for the simple rewards, Shannon utility, and Jensen-Shannon divergence are near-identical, which shows the technique's robustness.

\section{CONCLUDING REMARKS}

We have considered the task of data gathering through probing of an observed system; that is, affecting the system and monitoring its reactions. We have described a technique for evaluating potential probing actions, which is based on a combination of the Bayesian reasoning, a generalized utility model, and a conservative heuristic for using multiple observations without knowing their joint distribution.

The initial tests within the PAINT architecture have confirmed that this technique selects the right probes and gives accurate hypothesis-evaluation results, but the context of these tests has been limited. We plan to evaluate it in more complex scenarios, including selection among a large number of hypotheses, processing a large volume of observations, and using of a wider range of utility functions. A longer-term challenge is to integrate it with Bayesian networks and develop more effective heuristics for dealing with observations that corroborate or contradict each other. 


\section{ACKNOWLEDGMENTS}

We are grateful to Helen Mukomel, Peter Brooks, and Nancy Roberts for their detailed comments and suggestions, which have helped to extend and focus the presentation.

\section{REFERENCES}

[Bardak et al., 2006a] Ulas Bardak, Eugene Fink, and Jaime G. Carbonell. Scheduling with uncertain resources: Representation and utility function. In Proceedings of the IEEE International Conference on Systems, Man, and Cybernetics, pages 1486-1492, 2006.

[Bardak et al., 2006b] Ulas Bardak, Eugene Fink, Chris R. Martens, and Jaime G. Carbonell. Scheduling with uncertain resources: Elicitation of additional data. In Proceedings of the IEEE International Conference on Systems, Man, and Cybernetics, pages 1493-1498, 2006.

[Fink et al., 2006] Eugene Fink, P. Matthew Jennings, Ulas Bardak, Jean Oh, Stephen F. Smith, and Jaime G. Carbonell. Scheduling with uncertain resources: Search for a near-optimal solution. In Proceedings of the IEEE International Conference on Systems, Man, and Cybernetics, pages 137-144, 2006.

[Fu et al., 2008] Bin Fu, Eugene Fink, and Jaime G. Carbonell. Analysis of uncertain data: Tools for representation and processing. In Proceedings of the IEEE International Conference on Systems, Man, and Cybernetics, 3256-3260, 2008.

[Gershman et al., 2009] Anatole Gershman, Eugene Fink, Bin $\mathrm{Fu}$, and Jaime G. Carbonell. Analysis of uncertain data: Evaluation of given hypotheses. In Proceedings of the IEEE International Conference on Systems, Man, and Cybernetics, 2009.

[Grünwald and Dawid, 2004] Peter D. Grünwald and A. Philip Dawid. Game theory, maximum entropy, minimum discrepancy, and robust Bayesian decision theory. Annals of Statistics, 32(4), pages 1367-1433, 2004.

[Kullback, 1987] The Kullback-Leibler distance. The American Statistician, 41, pages 340-341, 1987.

[Kullback and Leibler, 1951] Solomon Kullback and Richard A. Leibler. On information and sufficiency. Annals of Mathematical Statistics, 22, pages 79-86, 1951.

[Lin, 1991] Jianhua Lin. Divergence measures based on the Shannon entropy. IEEE Transactions on Information Theory, 37(1), pages 145-151, 1991.

[Shannon, 1948] Claude E. Shannon. A mathematical theory of communication. The Bell System Technical Journal, 27, pages 379-423 and 623-656, 1948.
TABLE I: DIFFERENTIATION BETWEEN TWO HYPOTHESES. WE HAVE EXPERIMENTED WITH FIVE PROBES AND FIVE TECHNIQUES FOR SELECTING RELEVANT OBSERVATIONS. FOR EACH PROBE AND EACH TECHNIQUE, WE HAVE RUN 2000 EXPERIMENTS, AND WE GIVE THE PERCENTAGE OF CORRECT HYPOTHESIS IDENTIFICATIONS.

\begin{tabular}{|c|ccccc|}
\hline $\begin{array}{c}\text { Probe } \\
\text { Num. }\end{array}$ & \multicolumn{6}{|c|}{$\begin{array}{c}\text { Simple } \\
\text { Reward }\end{array}$} & $\begin{array}{c}\text { Shannon } \\
\text { Utility }\end{array}$ & $\begin{array}{c}\text { Jensen- } \\
\text { Shannon }\end{array}$ & $\begin{array}{c}\text { Random } \\
\text { Observ. }\end{array}$ & $\begin{array}{c}\text { Indep. } \\
\text { Assum. }\end{array}$ \\
\hline \multicolumn{7}{|c|}{ Differentiating $\boldsymbol{H}_{\mathbf{1}}$ from $\boldsymbol{H}_{\mathbf{2}}$} \\
\hline $\mathbf{1}$ & 60 & 60 & 60 & 53 & 50 \\
$\mathbf{2}$ & 99 & 99 & 99 & 57 & 49 \\
$\mathbf{3}$ & 79 & 79 & 79 & 51 & 50 \\
$\mathbf{4}$ & 89 & 89 & 89 & 54 & 51 \\
$\mathbf{5}$ & 80 & 80 & 80 & 53 & 51 \\
\hline
\end{tabular}

\begin{tabular}{|l|lllll|}
\multicolumn{7}{c|}{ Differentiating $\boldsymbol{H}_{\mathbf{1}}$ from $\boldsymbol{H}_{\mathbf{3}}$} \\
\hline $\mathbf{1}$ & 84 & 84 & 84 & 54 & 49 \\
$\mathbf{2}$ & 99 & 99 & 99 & 60 & 50 \\
$\mathbf{3}$ & 79 & 79 & 79 & 52 & 50 \\
$\mathbf{4}$ & 89 & 89 & 89 & 54 & 50 \\
$\mathbf{5}$ & 85 & 84 & 84 & 55 & 49 \\
\hline
\end{tabular}

\begin{tabular}{|l|lllll|}
\multicolumn{7}{c|}{ Differentiating $\boldsymbol{H}_{\mathbf{1}}$ from $\boldsymbol{H}_{\mathbf{4}}$} \\
\hline $\mathbf{1}$ & 100 & 100 & 100 & 59 & 50 \\
$\mathbf{2}$ & 100 & 100 & 100 & 60 & 49 \\
$\mathbf{3}$ & 100 & 100 & 100 & 59 & 50 \\
$\mathbf{4}$ & 100 & 100 & 100 & 59 & 49 \\
$\mathbf{5}$ & 100 & 100 & 100 & 61 & 49 \\
\hline
\end{tabular}

TABle II: Differentiation aMong fOUR hyPOTHESES. We HAVE EXPERIMENTED WITH FIVE PROBES AND TWO UTILITY FUNCTIONS. FOR EACH PROBE AND EACH UTILITY, WE HAVE RUN 2000 EXPERIMENTS, AND WE GIVE THE PERCENTAGE OF CORRECT HYPOTHESIS IDENTIFICATIONS.

\begin{tabular}{|c|c|c|}
\hline \multirow{2}{*}{$\begin{array}{l}\text { Probe } \\
\text { Num. }\end{array}$} & \multicolumn{2}{|c|}{$\begin{array}{l}\text { Accuracy of hypothe- } \\
\text { sis selection (\%) }\end{array}$} \\
\hline & $\begin{array}{l}\text { Simple } \\
\text { Reward }\end{array}$ & $\begin{array}{l}\text { Shannon } \\
\text { Utility }\end{array}$ \\
\hline \multicolumn{3}{|c|}{$\begin{array}{c}\text { Differentiating } H_{1} \text { from the } \\
\text { other three hypotheses }\end{array}$} \\
\hline 1 & 77 & 76 \\
\hline 2 & 94 & 94 \\
\hline 3 & 86 & 86 \\
\hline 4 & 94 & 94 \\
\hline 5 & 89 & 89 \\
\hline
\end{tabular}

Selecting the right hypothesis

\begin{tabular}{|c|cc|} 
among $\boldsymbol{H}_{\mathbf{1}}, \boldsymbol{H}_{\mathbf{2}}, \boldsymbol{H}_{\mathbf{3}}$, and $\boldsymbol{H}_{\mathbf{4}}$ \\
\hline $\mathbf{1}$ & 50 & 49 \\
$\mathbf{2}$ & 61 & 49 \\
$\mathbf{3}$ & 47 & 50 \\
$\mathbf{4}$ & 49 & 50 \\
$\mathbf{5}$ & 50 & 50 \\
\hline
\end{tabular}

